\title{
SMDP - a Simple Multimedia Service Description Protocol on the Internet
}

\author{
Qiang Nelson Yin Chung-Horng Lung \\ Department of Systems and Computer Engineering \\ Carleton University, Ottawa, Canada \\ \{nqyin, chlung\}@sce.carleton.ca
}

\begin{abstract}
Downloading multimedia content from the Internet becomes more and more popular. Consumers have more to consider than just the name of the artist or the title of the content when they buy online. Based on the current model, it is time consuming for consumers to obtain the desired multimedia content, starting from the beginning of searching a title to the end of placing a purchase order. We have proposed a protocol MSAP (Multimedia Service Announcement Protocol) that enables consumers to search and download multimedia content more effectively and faster. This paper builds on the concept of MSAP for content search and distribution. This paper presents SMDP (Simple Multimedia service Description Protocol) that is used for multimedia description for both the service and content levels. SMDP can be used for service providers to effectively convey multimedia information to the consumers.
\end{abstract}

\section{Introduction}

For an Internet user, it seems that it is very easy to download music or movie from the Internet. All they have to do is to run their Internet browser and use Multimedia Search Engine, such as Singingfish [2], to search multimedia contents. Reference [3] pointed out the abilities of a typical multimedia search engine. However, the Internet also creates new challenges. The biggest problem facing users of web search engines today is the quality and quantity of the results they get. The search is heavily dependent on the algorithms that typically rely on keyword matching which usually return too many low quality matches [1]. For instance, to search "Time to say goodbye" sung by Andrew Bocelli, using Singingfish in our experiments, we obtained 581,320 returned items and only one of them is instantly downloadable [8].

It is very time consuming for users to obtain the multimedia content, because the search engine does not verify the authenticity or accuracy at all. In addition, many results only provide links to the providers' web sites, from which the user has to go through yet another screening process or purchasing process in order to purchase or download multimedia content. One approach to mitigate the problem is to enhance the search algorithm. However, the fundamental challenge is that the content are scattered all over the Internet and there are a large number of possible variabilities, e.g., content providers, identical or similar titles, multimedia formats, and so on. Other issues include the order of the results presented by search engines [4] and they do not have pricing and content provider's information.

On the Internet, a number of content providers and content distributors are providing multimedia service. MSAP (Multimedia Service Announcement Protocol) [8] was proposed to advertise multimedia service information and communicate the service information and multimedia content-specific information necessary for online multimedia content producers, distributors and consumers. MSAP can be used extensively for multimedia service advertisement.

To obtain multimedia services, a user has to know the information of service providers who are providing relevant services and the content. Service descriptions assist the advertisement of those services and convey the relevant setup information to prospective users. SMDP is designed to convey such information to recipients. SMDP is purely a format for service description - it does not incorporate a transport protocol, and is intended to use MSAP or other different transport protocols such SIP (Session Initiation Protocol) [5], RTSP (Real-Time Streaming Protocol) [7], or the HTTP (Hypertext Transport Protocol). SMDP is intended to be general purpose, so that it can be used for a wider range of network environments and applications.

\section{SMDP and MSAP}

SMDP is a description protocol for MSAP [8]. MSAP is used for MSPs (Multimedia Service Providers) client to announce its presence and the services they provide by periodically multicasting an announcement packet to a well known multicast address and port using the MSAP. Figure 1 illustrates the architecture of MSAP. 


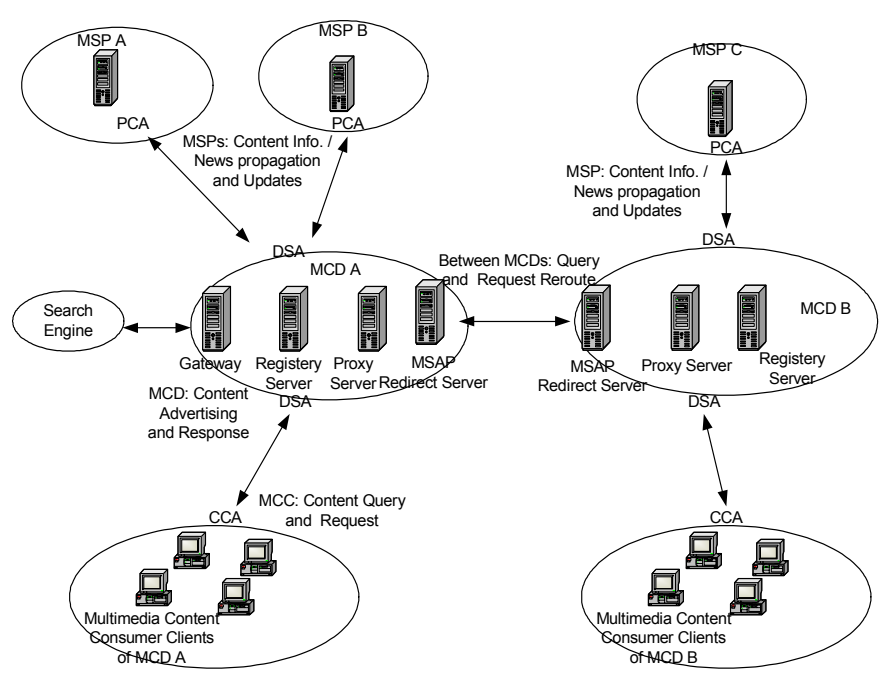

Figure 1. Architecture of MSAP

SMDP serves two primary purposes:

1) It is a means for MSPs to describe the service they provide and to communicate the existence of an MCC (Multimedia Content Consumer); and

2) It is a means for MCDs (Multimedia Content Distributors) to convey sufficient information to enable MCCs to obtain the service and content.

Figure 2 shows the application of MSAP and SMDP.

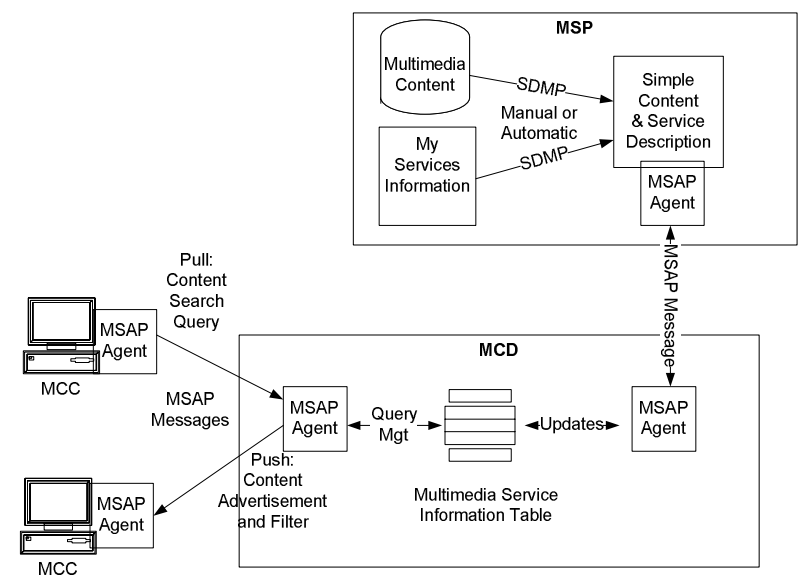

Figure 2. Using MSAP and SDMP for Multimedia Content Distribution

MSAP uses UDP with the following format:

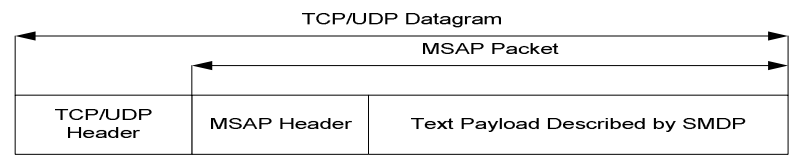

Figure 3. MSAP Packet Containing SMDP

The description of SMDP typically includes:

1) MSP's information (Service provider's name, MSAP domain name, IP address, MSAP distributor ID)
2) Service types (Real-time online streaming and nonreal-time download)

3) Time(s) that the service is active

4) The media content that is available for service. (Title name, formats, size and etc.)

5) Information to receive those multimedia content (server addresses, ports, protocol and so on)

6) Additional information may be desirable, as users to obtain a service may have limited or specific needs:

- Information about the bandwidth to be used by each recipient of service to achieve the QoS (Quality of Service) requirement.

- Information about the usage of the service.

- Contact information for the person who is responsible for the content distribution.

In general, SMDP must convey sufficient information for MSP to announce the service and resources to be used by potential participants who may need to know and to serve the MCCs.

\section{SMDP Two-Level Descriptions}

An SMDP description consists of a number of lines of text of the form $<$ type $>=<$ value $>$. $<$ type $>$ is character and is case sensitive. $<$ value $>$ is a structured text string whose format depends on <type $>$. It is also casesignificant unless a specific field defines otherwise.

As shown in Figure 4, an MSAP service announcement consists of a SMDP service-level description followed by zero or more SMDP contentlevel descriptions. An SMDP service-level description consists of details that apply to the whole service and all multimedia content. On the other hand, the optional content-level description, are those details that apply to a single multimedia content. The service-level part starts with an 'sl=' line and continues to the first multimedialevel section. The content-level description starts with a ' $\mathrm{cl}=$ ' line and continues to the next multimedia description or end of the whole service description.

When SMDP is conveyed by MSAP, only one service description is allowed per packet. When SMDP is conveyed by other means, many SMDP service descriptions may be concatenated together (the 'sl=' line indicates the start of a service description which also terminates the previous description). Some lines in each description are required and some are optional but all must appear in exactly the order given here (the fixed order greatly enhances error detection and allows for a simple parser). Optional items are marked with an '*'.

SMDP descriptions are entirely textual using the ISO 10646 character set in UTF-8 encoding. SMDP field names and attributes names use only the US-ASCII subset of UTF-8, but textual fields and attribute values may use the full ISO 10646 character set. 


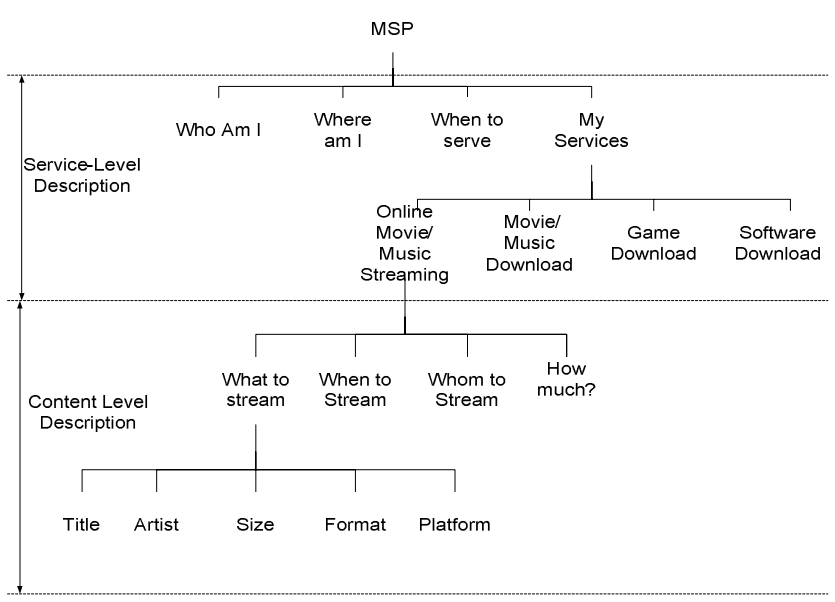

Figure 4. SMDP Two-Level Descriptions

The textual form was chosen, because: (i) It enhances portability and enables a variety of transports to be used and to allow flexible, text-based toolkits to be used to generate and process service descriptions; (ii) It saves the bandwidth for MSAP announcements; and (iii) It is easy to detect most errors, since the encoding was designed with strict order and formatting rules. An MSAP announcement may be transported via unreliable means or damaged by an intermediate proxy server. This allows discarding of encrypted announcements for which a receiver does not have the correct key.

\subsection{Service-Level Description}

Table 1 summarizes the MSAP service level description. As shown in Table 1, a MSAP service-level description contains seven types of description:

Protocol Description. The "v" field gives the version of the SMDP. SMDP starts with version 0.

MSP Information. In general, the "so=" field serves as a globally unique identifier of the MSP description. so $=<$ MSPusername $><$ announcement $\mathrm{id}><$ address $>$.

The "so =" field gives the service originator of their MSAP name and the IP address plus a session id which is used to identify the session connecting to the MCD proxy server and session version number. It is needed for proxy server announcements to detect which of several announcements for the same session is the most recent. <address $>$ is the IP address. In version 0, only IP4 is used. The IP address can be the fully qualified domain name or the dotted-decimal representation.

The $\mathrm{su}=$ field is the Universal Resource Identifier (URI) as used by WWW clients. The URI should be a pointer to additional information about a MSP.

MSP Contact Information. This specifies contact information for the MSP. It includes information such as $\mathrm{se}=<$ email address $>$ and $\mathrm{sp}=<$ phone number $>$.
Table 1. MSAP Service Level Description

\begin{tabular}{|c|c|}
\hline Types & Fields and Sub-fields \\
\hline $\begin{array}{l}\text { Protocol } \\
\text { description }\end{array}$ & $\mathrm{v}=\langle 0\rangle$ \\
\hline MSP info & $\begin{array}{l}\mathrm{so}=<\text { MSP username }><\text { announcement } \mathrm{id}><\text { addr. }> \\
\mathrm{su}=<\mathrm{URI}>\end{array}$ \\
\hline $\begin{array}{l}\text { MSP contact } \\
\text { information }\end{array}$ & $\begin{array}{l}\mathrm{se}=<\text { email address }> \\
\mathrm{sp}=<\text { phone number }>\end{array}$ \\
\hline MSP Service info & $\begin{array}{l}\mathrm{sn}=\mathrm{RTCS} / \mathrm{NRTCD} \\
\mathrm{si}=<\text { description }>\end{array}$ \\
\hline $\begin{array}{l}\text { Announcement } \\
\text { Connection info }\end{array}$ & $\begin{array}{l}\mathrm{ac}=<\text { MSAP addr. }><\text { protocol }><\text { connection } \\
\text { addr } .><\text { session id }>\end{array}$ \\
\hline Time & $\begin{array}{l}\text { at }=<\text { announcement start time }><\text { announcement } \\
\quad \text { service stop time }> \\
\text { ar }=<\text { repeat interval }><\text { active duration }>\end{array}$ \\
\hline Encryption Keys & $\mathrm{k}=$ uri:<encryption key $>$ \\
\hline
\end{tabular}

Phone numbers should be given in the conventional international format - preceded by a "+ and the international country code. There must be a space or a hyphen ("-") between the country code. For example: $\mathrm{p}=+1-613-520-2600$ or $\mathrm{p}=+1867703303601$.

MSP Service information. The "sn=" field is the service name. For SMDP version 1, there are two types of services, RTCS is defined for real-time content streaming and NRTCD for non-real-time download.

The "si $=<$ description $>$ " field contains a short description about the general information of the entire services provided by an MSP. For example, the total number of music titles that is available for service.

$s b$ attribute specifies the proposed bandwidth to be used by the service session between MCC and MSP. sb $=<$ modifier $>$ : $<$ bandwidth-value $>$. $<$ modifier $>$ is a single alphanumeric word giving the meaning of the bandwidth value. Three modifiers are initially defined:

1)Total: the maximum bandwidth associated with all connection with proxy servers in one or more MSAP distribution domains.

2)CS: Connection-Specific: the maximum bandwidth for each connection between an agent and the server.

3)AS: the maximum bandwidth for a specific announcement, e.g., a multimedia server update.

Announcement Connection Information. This field includes the connection information for an MSP to connect an MSAP proxy server. The "ac =" field contains connection data. ac $=<$ MSAP address $>$ $<$ protocol $><$ connection addr. $><$ session id $>$. An MSAP announcement must contain one "ac $="$ field in each description (see below) or an "ac $="$ field at the contentlevel. The first sub-field is the MSP MSAP ID. The second sub-field is <protocol> which is the transport protocol, UDP in SMDP version 1, used to set up the connection. The third sub-field is the connection IPv4 address, which is followed by an announcement session id used to identify the session connecting to the server.

Since an MSAP agent uses an IP multicast or specified unicast address to discover the proxy server, typically 
the connection address could be a class-D IP multicast group address or IP unicast address.

Announcement time descriptions. at = $<$ announcement start time $><$ announcement service stop time $>$. The field specifies the start and stop times for an MSP's announcement. It can be considered as a session's start and stop time between an MSP and an MCD. These values are represented using Network Time Protocol (NTP) times. To convert these values to UNIX time, subtract 2208988800 . If the stop time is zero, then the session is not bounded, though it will not become active until after the start time. If the start time is also zero, the service is regarded as permanent.

The "ar=" field specifies repeat times for an MSAP announcement between an MSP and an MCD using the format ar $=<$ repeat interval $><$ active duration $>$. For example, if a session is active at 10am on Monday and $11 \mathrm{am}$ on Tuesday for one hour each week for three months, then the <start time $>$ in the corresponding "at=" field would be the NTP representation of 10am on the first Monday, the <repeat interval $>$ would be 1 week, the $<$ active duration $>$ would be 1 hour. The corresponding stop time of the "at=" field would be the NTP representation of the end of the last session three months later. By default all fields are in seconds, so the "ar =" and "at=" fields might be:

- at $=0$

- $\operatorname{ar}=6048003600090000$

Both at and ar could also be written in a form of $d d$ (the number of days) $h h$ (the number of hours). So for the last example: at $=0$; $\operatorname{ar}=7 \mathrm{~d} 1 \mathrm{~h}$

Encryption Keys. SMDP may be used to convey encryption keys. A key field is permitted before the first content entry. The format is $\mathrm{k}=<\operatorname{method}>:<$ encryption key $>$. The method indicates the mechanism to be used to obtain a valid key by external means or from the encoded encryption key given.

$\mathrm{k}=$ uri: $<$ URI to obtain key $>$ : A Universal Resource Identifier as used by WWW clients. The URI refers to the data containing the key, and may require additional authentication.

Attributes are the primary means for extending SMDP. Attributes may be used service-level, content-level, or both. Their names must be in the US-ASCII subset of ISO-10646/UTF-8. A content description may have any number of attributes ("a =" fields), which are content specific. These are referred to as "content-level" attributes which add information to the content. Attribute fields can also be added before the first content field. The service-level attributes convey additional information that applies to the entire MSP service instead of an individual multimedia content. An example could be the special service announcement.

The following provides an example of SMDP servicelevel description: A MSP, filmlinks, whose URL is $w w w$.filmlink.com, is providing audio and video content and real-time video on demand service. It has more than 1000 movie titles and 1,000,000 music titles. The email and the contact number of filmlinks are web@filmlinks.com and +1 $613 \quad 234$ 5678. The announcements from MSP to MCD are permanent. And announcement will be once per hour and the proxy server $(10.10 .10 .10)$ will be connected by using UDP and the session id is 2543 .

The corresponding SDMP descriptions are as follows:

$\mathrm{v}=0$

so=filmlinks.com@bell.ca 12345678 IP4 126.16.64.4

$\mathrm{su}=<w w w$. filmlinks.com $>$

$\mathrm{se}=$ web@filmlinks.com

$\mathrm{sp}=<+16132345678>$

$\mathrm{sn}=<\mathrm{rtcs}, \mathrm{nrtcd}>$

$\mathrm{si}=<$ filmlinks has more than 1000 movie titles and

$1,000,000$ music titles $>$

at $=0$

ar $=0 \mathrm{~d} 1 \mathrm{~h}$

ac=<filmlinks@bell.ca $><$ udp $><10.10 .10 .10><2543>$

\subsection{Content-Level Description}

This section discusses the content-level description used in the SMDP. An SMDP description may contain a number of content-level descriptions, shown in Table 2.

Table 2. MSAP Content-Level Descriptions

\begin{tabular}{|c|c|}
\hline Types & Fields \\
\hline $\begin{array}{l}\text { Multimedia } \\
\text { Content }\end{array}$ & $\begin{aligned} & \mathrm{mc}=<\text { type }><\text { title }><\text { size }><\text { format }><\text { language }> \\
&<\text { length }><\text { date }> \\
& \mathrm{mp}=<\text { method }><\text { currency value }>\end{aligned}$ \\
\hline $\begin{array}{l}\text { Producer } \\
\text { Distributor info }\end{array}$ & $\begin{array}{l}\text { pi }=<\text { name }> \\
\text { pu }=<\text { URI }>\end{array}$ \\
\hline $\begin{array}{l}\text { Service } \\
\text { Connections info }\end{array}$ & $\begin{array}{l}\mathrm{sc}=<\text { transport }><\text { addr }><\text { port }><\text { username }> \\
<\text { password }> \\
\mathrm{sb}=<\text { modifier }>:<\text { bandwidth-value }>\end{array}$ \\
\hline Service Time & svt $=<$ service type $><$ start time $><$ stop time $>$ \\
\hline Security & $\mathrm{k}=<$ method $>:<$ encryption key $>$ \\
\hline $\begin{array}{l}\text { Other Suggested } \\
\text { Attributes }\end{array}$ & $\begin{array}{l}\mathrm{a}=\text { Alb: }<\text { Album }>\text {; }=\text { charset }:<\text { character } \text { set }> \\
\mathrm{a}=\text { art: }<\text { Artists }>\text {; } \mathrm{a}=\text { poster }:<\text { posterURI }> \\
\mathrm{a}=\text { rate }:<\text { rate }>\text {; } \mathrm{a}=\text { framerate }:<\text { frame rate }>\end{array}$ \\
\hline
\end{tabular}

The following illustrates each field listed in the table:

Multimedia Content Fields. A multimedia content field has several sub-fields:

1)The first sub-field is the multimedia type, audio or video. The list may be extended to electronic game.

2) The second sub-filed is multiple alphanumeric words showing the title for a content.

3) The size of content is indicated in size value subfield. The value is in kilobyte.

4) The fourth and subsequent sub-fields are content formats. For audio and video, these will normally be a multimedia payload type as defined in the RTP Audio/Video Profile. Typical format types include: WMV, RM, AVI, MP2, MPEG2 [6]. 
5) The language sub-field specifies the default language. If multiple languages are provided at the content level, a multiple-language attribute can be defined and the order indicates the order of importance.

6) The sixth sub-field is the length of time and it is in the format of hh (hours): mm (minutes): $\sec ($ seconds) to show the total time length of the content.

7) The seventh sub-field indicates the release date for the content using yyyy(year):mm(month):dd(date).

$m p$ field provides the pricing information for the content. The format is $\mathrm{mp}=<$ method $><$ currency-value $>$. The first sub-field defines means to pay for the content: free $=$ free, $p p v=$ pay per view, $\operatorname{mbs}=$ membership, $p c h$ $=$ purchase. The second field specifies the currency and the value of price, e.g. US $\$ 0.5$

Content Producer or Distributor's Information. This field specifies contact information for the content producer or distributor. It includes field name $=$ (the name of producer or distributor) and the phone field $\mathrm{p}=*$ (phone number). The $\mathrm{u}$ field is a Universal Resource Identifier as used by WWW clients and it is a pointer to additional information about a MSP/MCP.

Service Connection Information. This includes the service connection information for each MCC to communicate with the MSP and obtain the service.

1) $\mathrm{sc}$, "sc $=<$ transport $><$ address $><$ port $><$ username $>$ $<$ password $>$ ". The first sub-field is the protocol used for MCC to set up a connection with MSP. Those protocols include UDP, SIP (Session Initiation Protocol), RTSP (Real-Time Streaming Protocol), and HTTP.

2) The second sub-field is the IPv4 address. It is followed by the transport port to which the multimedia content will be dispatched.

3) The third sub-field is the transport ports to carry the multimedia stream. Depending on the applications, it may be necessary to specify multiple transport ports or the transport protocol and format separately.

4) The fourth and fifth sub-fields are the username and password for the clients to access the MSP service server or participate in a secure streaming session.

The $s b$ field specifies the proposed bandwidth to be used between MCCs and the MSP. sb $=<$ modifier $>$ : $<$ bandwidth-value $>$, which is similar to MSP Service Information. Two modifiers are initially defined:

1) $t b$ : the maximum bandwidth associated with all connections for an MSP's server, e.g., the VoD server.

2) $c s$ (Connection-Specific): the maximum bandwidth for each connection between a MCC and the MSP.

Service Time information. $s t=<$ service start time $>$ $<$ service stop time $>\quad$ "st $="$ field specifies the start and stop times for an MSP's service. Unlike the announcement time, these values are presented in the form of yyyy(year):mm(month):dd(date):hh(hours):mm (minute):sec(second). For real-time services like video streaming, service start and stop time must be specified.
For non-real-time service, if the start-time is zero, the service is regarded as permanent.

Encryption Keys. Like service-level description, the content level description also can contain encryption keys. The main difference between those two levels is that generally the encryption for service level is the security of communication channel, but the content level is for the digital right management.

Similarly, the key field in content level description $\mathrm{k}=$ $<$ method $>$ :<encryption key $>$. The method sub-field indicates the mechanism used to obtain a usable key by external means or from the encoded encryption key.

Other Suggested Attributes. The following attributes are suggested for content-level description.

1) $\mathrm{a}=\mathrm{Alb}:<$ Album $>$. The album attribute is only used for music titles that belong to a published album.

2) $a=$ art: $<$ Artists $>$. This may have multiple words indicating the singer(s) or actor/actress.

3) $a=$ rate: $<$ rate $>$. Every country has its own standards to classify movie. This attribute gives the classification category of the movie and it is only used for movie.

4) $a=$ charset: $<$ character set $>$. This specifies the character set used to display the content name and information. By default, the ISO-10646 character set in UTF-8 encoding is used. Other more compact representation, such as ISO-8859-1, may be used.

5) $\mathrm{a}=$ poster: $<$ posterURI $>$. A Universal Resource Identifier refers to the web site containing posters or description of the multimedia title.

6) $\mathrm{a}=$ framerate: $<$ frame rate $>$. This is the maximum video frame rate in frames/sec. It is intended as a recommendation for the encoding of video data.

\subsection{A SMPD Example}

This section provides an example of the SMDP content level description: An MSP, filmlinks ( $w w w$. filmlink.com), is about to stream a movie "The Day after Tomorrow" at 8:00pm on December 102004 and the service will stop at 11:00pm. The movie is in English, is about 90 minutes, and is free for members.

The movie is in Window Media format. The size of the movie is $500 \mathrm{Mb}$. In order to watch the movie, MSAP clients must use the RSTP protocol and the following address: RTSP: 10.10.10.10:32, and follow MSAP username-filmlink and password-asbcdef, to access the filmlinks' VoD server. The movie producer is Warner Brothers and the release date is May $30^{\text {th }}, 2004$. The recommended bandwidth for MCC users is $300 \mathrm{kbs}$. This link (www.filmlink.com/movie/todayaftertomorrow_ptr. jpeg) provides a few posters and snapshots of the movie and the movie illustrates the natural disasters could happen to earth in the near future if global environment is not under protection. Director of this movie is John and the main actor and actress is Luc and Gord. 3311697600 is converted to Dec 10, 2004 8pm and 
3311708400 is converted to Dec 10, $200411 \mathrm{pm}$. The corresponding SDMP descriptions are as follows: $\mathrm{mc}=<$ video $><$ The day after tomorrow $><500 \mathrm{Mb}>$

$<$ wmv $><$ english $><1$ h30m $><20040530>$

$\mathrm{mp}=$ free 0

$\mathrm{pi}=<$ warner brothers $>$

$\mathrm{pu}=<$ www.hollywood.cm $>$

$\mathrm{sc}=<$ rstp $><10.10 .10 .10><32><$ filmlink $><$ asbcdef $>$

$\mathrm{sb}=<\mathrm{cs}>:<300>$

$\mathrm{svt}=<3311697600><3311708400>$

$\mathrm{a}=$ art: $<$ luc gord $>$

$\mathrm{a}=$ poster: $<$ www.filmlink.com/movie/todayaftertomorrow _ptr.jpeg $>$

\section{Implementation}

The implementation of the entire protocol is divided into a few stages. At the current stage, we are developing the prototype of MSAP and some simple test cases to verify the feasibility and functionality of MSAP. The test bed which consists of three Alcatel ATM switches/virtual routers, NMS (Network Management System), and a 3Com switch. Two servers simulating MSP and MCD are connecting two switches on each side of the network. Each server contains a database of thousands of music and movie entries. They are propagated to the MCD proxy server.

A group of PCs representing MCCs send out search quires and then request multimedia contents. Each MCC is provisioned with $200-300 \mathrm{Kbps}$ bandwidth. The search results responded by MCD server will displayed in the MST (Multimedia Service Table) at MCC agent's user interaction GUI.

At the next stage, we are going to compare the performance between search engine and MASP from the user perspective and also the scalability and robustness of MSAP. We plan to increase the size of MST and the database of MSP and generate queries in thousands from the MCC agent simultaneously and also add one more MCD proxy server to test the redirection of query. In addition, we are adapting the format using XML to be more flexible and compatible with other applications.

\section{Summary}

This paper proposed a protocol, SMDP, to support the MSAP architecture for multimedia content search and distribution. MSAP can significantly shorten the content searching time of multimedia content compared with the current model. SMDP was proposed to convey the multimedia sufficient information for multimedia service providers to announce the service and resources. The information will then be used by potential participants who may need to know and to serve the customers. SMDP was discussed in details in this paper.

We are developing the initial prototype and setting up a test bed to implement it. Our implementations already verified the feasibility of MSAP, the accuracy and reliability to deliver query results to MCCs with very low Internet access rates. And the download process has been shortened to 3 steps and no longer than 5 minutes. We are integrating SMDP into the MSAP architecture.

\section{References}

[1] S. Brin and L. Page, "The Anatomy of a Large-Scale Hypertextual Web Search Engine", http://wwwdb.stanford.edu/ backrub/google.html, Comp. Sci. Dept., Stanford Univ., 2002, accessed May 2005.

[2] www.singingfish.com

[3] http://www.marketleap.com/singingfish

[4] B. Comings, "Achieving Good Placement in Search Engine Results", http://www.spyrock.com/, 2002.

[5] M. Handley, et al., "SIP: Session Initiation Protocol", RFC 2543 , www.ietf.org, March 1999.

[6] B. S. Manjunath, et al., Introduction to MPEG 7: Multimedia Content Description Language, John Wiley \& Sons, 2002.

[7] H. Schulzrinne, et al., "Real Time Streaming Protocol", RFC 2326, www.ietf.org, April 1998.

[8] Q. N. Yin and C.-H. Lung, "A Protocol for Efficient Multimedia Search and Distribution on the Internet", Proc. of the 1st IEEE Int'l Workshop on Networking Issues in Multimedia Entertainment (NIME), in conjunction with IEEE GLOBECOM, Oct. 2004. 